\title{
Relação entre classe funcional e fração de ejeção do ventrículo esquerdo em pacientes com doença coronariana candidatos à reabilitação cardíaca
}

\author{
Relationship between functional class and left ventricular ejection fraction in patients \\ with coronary heart disease who were candidates for cardiac rehabilitation
}

\author{
Andrieli Barbieri Garlet ${ }^{1}$, Dannuey Machado Cardoso ${ }^{2}$, Tamires Daros dos Santos ${ }^{3}$, Sérgio Nunes Pereira ${ }^{4}$, \\ Isabella Martins de Albuquerque ${ }^{5} \bowtie$



\section{RESUMO}

OBJETIVOS: Avaliar a potencial relação entre classe funcional segundo a New York Heart Association e a fração de ejeção do ventrículo esquerdo em pacientes com doença coronariana candidatos à reabilitação cardíaca.

MÉTODOS: Foi realizado um estudo transversal retrospectivo, por meio da análise de prontuários de pacientes com doença coronariana candidatos ao Programa de Reabilitação Cardíaca do Hospital Universitário de Santa Maria, Rio Grande do Sul. Foram incluídos pacientes de ambos os sexos, com idade entre 50 e 65 anos, sendo excluídos aqueles com insuficiência renal crônica, anemia, ecocardiograma com baixa qualidade de imagem, pacientes com ritmo não sinusal e indivíduos cujos prontuários não apresentavam informações suficientes. A coleta dos dados ocorreu no período de agosto de 2015 a março de 2016, tendo sido extraídas dos prontuários as informações que compuseram as variáveis de interesse, tais como: dados clínicos e demográficos (sexo, idade, comorbidades, diagnóstico clínico, tratamento cirúrgico e medicamentoso), valores da fração de ejeção do ventrículo esquerdo coletados através de ecocardiografia (Doppler convencional e Doppler tecidual) e a classe funcional a partir do teste ergométrico. A análise estatística foi realizada mediante a aplicação do teste de Kruskal-Wallis seguido do post hoc de Dunn. O nível de significância estatística adotado foi de $\mathrm{p}<0,05$.

RESULTADOS: Foram avaliados consecutivamente 131 prontuários, sendo que 102 preencheram os critérios de inclusão. A média de idade da amostra foi de 59,23 7,95 anos e $70(68,63 \%)$ pacientes eram do sexo masculino. Ocorreu predomínio da classe funcional I. Observouse relação inversa entre classe funcional e fração de ejeção do ventrículo esquerdo: quanto mais avançada a classe funcional, maior foi o comprometimento do desempenho cardíaco $(\mathrm{p}=0,036)$.

CONCLUSÕES: Nesta amostra de pacientes com doença coronariana, ingressantes em um programa de reabilitação cardíaca, houve relação inversa entre fração de ejeção do ventrículo esquerdo e progressão da classe funcional. Este achado fornece informações sobre as limitações impostas pela doença na capacidade de exercício e na função cardíaca do paciente e pode auxiliar na elaboração de um programa de treinamento físico.

DESCRITORES: cardiopatia coronariana; revascularização miocárdica; angioplastia; reabilitação.

\section{ABSTRACT}

AIMS: To assess the potential relation between the New York Heart Association functional class and left ventricular ejection fraction in coronary heart disease patients who were candidates for cardiac rehabilitation.

METHODS: This is a retrospective cross-sectional study based on the analysis of medical records of coronary heart disease patients who were candidates for the Cardiac Rehabilitation Program of Hospital Universitário de Santa Maria, state of Rio Grande do Sul, Brazil. Both male and female patients aged 50 to 65 were included, whereas patients with chronic renal failure, anemia, poor echocardiographic image quality, nonsinus rhythm, and also those individuals whose medical records lacked sufficient information were excluded. Data were collected from August 2015 to March 2016, and the information that made up the variables of interest was extracted from the medical records, such as: clinical and demographic data (sex, age, comorbidities, clinical diagnosis, surgical and drug treatment), left ventricular ejection fraction values obtained by echocardiography (conventional and tissue Doppler echocardiography), and functional class from the ergometric test. Statistical analysis was performed using Kruskal-Wallis test followed by Dunn's post-hoc test. The significance level was set at $\mathrm{p}<0.05$.

RESULTS: A total of 131 medical records were consecutively evaluated, of which 102 met the inclusion criteria. Mean age was $59.23 \pm 7.95$ years and $70(68.63 \%)$ patients were male, with a predominance of functional class I. There was an inverse relation between functional class and ejection fraction: the more advanced the functional class, the more compromised the cardiac performance $(\mathrm{p}=0.036)$.

CONCLUSIONS: In this sample of patients with coronary heart disease who were candidates for cardiac rehabilitation, there was an inverse relation between left ventricular ejection fraction and functional class. This finding provides information about the limitations imposed by the disease on patient's exercise capacity and heart function and can contribute to the development of a physical training program.

KEY WORDS: coronary heart disease; myocardial revascularization; angioplasty; rehabilitation. 
Abreviaturas: FEVE, fração de ejeção do ventrículo esquerdo; NYHA, New York Heart Association; RC, reabilitação cardíaca.

\section{INTRODUÇÃO}

O envelhecimento populacional consequente ao aumento progressivo na expectativa de vida vem ocasionando elevação dos índices de mortes e internações por causas cardiovasculares [1]. As quatro doenças crônicas não transmissíveis de maior impacto mundial compreendem doenças cardiovasculares, diabetes, câncer e doenças respiratórias crônicas. Dentre estas, as doenças cardiovasculares correspondem às principais causas de morte e invalidez no Brasil e no mundo [2].

Após um evento cardíaco é necessária uma abordagem multidisciplinar para o aconselhamento na prevenção da reincidência de episódios, melhorando a adesão ao tratamento medicamentoso e estimulando mudanças no estilo de vida [3]. Diante desse cenário, a reabilitação cardíaca (RC) apresenta-se como uma ferramenta não farmacológica que contribuiu para minimizar os efeitos fisiopatológicos e psicológicos das doenças cardiovasculares, além de modificar os fatores de risco apresentados pelos pacientes com doença coronariana [4].

O teste ergométrico é indicado para pacientes candidatos a programas de $\mathrm{RC}$, pois visa identificar a resposta eletrocardiográfica, avaliar a reação cronotrópica e pressórica após o esforço e definir o prognóstico da doença pela estratificação das classes funcionais segundo a New York Heart Association (NYHA) [5].

Outro parâmetro relevante na avaliação cardiovascular consiste no exame ecocardiográfico, o qual fornece informações imediatas sobre as câmaras cardíacas, a função sistólica e diastólica, a fração de ejeção do ventrículo esquerdo (FEVE) e outras variáveis [6]. Estudos demostram que em pacientes após infarto agudo do miocárdio ocorrem modificações na mecânica cardíaca, as quais podem ocasionar insuficiência cardíaca, devido à dilatação do ventrículo esquerdo e redução da FEVE, com impacto na classe funcional $[7,8]$.

$\mathrm{Na}$ prevenção secundária, a redução expressiva de eventos cardiovasculares poderia ser alcançada no momento em que os pacientes fossem submetidos a um programa de triagem e acompanhamento dos fatores de risco [9]. Assim, a estratificação de risco quanto à classe funcional segundo a NYHA, pelo teste ergométrico, e a mensuração da FEVE pela ecocardiografia, podem propiciar uma abordagem individualizada dos sujeitos ingressantes em um programa de $\mathrm{RC}$, fornecendo informações sobre as limitações impostas pela doença na capacidade funcional e na função cardiovascular do paciente. Essas informações podem auxiliar no estabelecimento de estratégias de RC, tais como o tipo de treinamento físico a ser empregado, otimizando os resultados clínicos obtidos durante e após a reabilitação.

Diante do exposto, o objetivo do presente estudo foi avaliar a potencial relação entre classe funcional e FEVE em pacientes com doença coronariana candidatos à RC.

\section{MÉTODOS}

Este estudo é parte de um projeto maior intitulado "Efeitos de um programa de reabilitação cardíaca (Fase II) pós-cirurgia de revascularização do miocárdio na tolerância ao exercício e biomarcadores laboratoriais" tendo sido aprovado pelo Comitê de Ética em Pesquisa da Universidade Federal de Santa Maria, sob o número 16149813.3.0000.5346. Trata-se de estudo transversal e retrospectivo, realizado por meio da análise de prontuários de pacientes com doença coronariana candidatos ao Programa de Reabilitação Cardíaca do Ambulatório de Cardiologia do Hospital Universitário de Santa Maria (HUSM). Os dados coletados foram do período de março de 2012 a março de 2016.

Foram incluídos pacientes de ambos os sexos, com idade entre 50 e 65 anos, sendo excluídos aqueles com insuficiência renal crônica, anemia, ecocardiograma com baixa qualidade de imagem, pacientes com ritmo não sinusal e indivíduos cujos prontuários não apresentavam informações suficientes.

A coleta de dados ocorreu no período de agosto de 2015 a março de 2016, tendo sido extraídas dos prontuários as informações que compuseram as variáveis de interesse: dados clínicos e demográficos (sexo, idade, comorbidades, diagnóstico clínico, tratamento cirúrgico e medicamentoso), valores da FEVE coletados através de ecocardiografia (Doppler convencional e Doppler tecidual) e a classe funcional a partir do teste ergométrico.

A quantificação objetiva da classe funcional foi realizada mediante informações obtidas a partir do teste ergométrico, conforme classificação da NYHA, que considera quatro categorias, com grau de limitação crescente: I) sem limitações nas atividades; II) presença de dispneia em atividades físicas comuns; III) com dispneia ao mínimo esforço; e IV) presença de dispneia mesmo em repouso [5].

O cálculo do tamanho da amostra foi realizado com base em um estudo piloto com os primeiros 10 
pacientes incluídos no estudo, onde para detectar uma relação significativa entre a FEVE e a classe funcional com um $\mathrm{r}^{2}$ de 0,067 , poder de $80 \%$, alfa de $5 \%$ e acrescido de $10 \%$ para perdas, foi necessária a inclusão mínima de 97 pacientes no estudo.

Os dados foram analisados através do programa IBM SPSS Statistics (versão 20.0) e avaliados quanto à normalidade pelo teste de Kolmogorov-Smirnov. Os dados categóricos foram apresentados em frequências absolutas e porcentagens. Os dados contínuos com distribuição normal foram apresentados em média e desvio padrão. Para verificar a relação entre classe funcional e FEVE foi utilizado o teste de KruskalWallis seguido do post hoc de Dunn. O nível de significância foi estabelecido em $\mathrm{p}<0,05$.

\section{RESULTADOS}

Foram analisados consecutivamente os prontuários de 131 pacientes de ambos os sexos, candidatos à $\mathrm{RC}$, com idade entre 50 e 65 anos e submetidos à angioplastia (com ou sem implante de stent) ou revascularização do miocárdio. Foram excluídos 29 prontuários, pois não apresentavam informações suficientes.

A amostra foi constituída por 102 pacientes, dos quais $68,63 \%(\mathrm{n}=70)$ eram do sexo masculino, sendo a média de idade de 59,23 $\pm 7,95$ anos. Considerando o índice de massa corporal, observou-se que 31 pacientes (30,39\%) apresentavam peso normal, 39 (38,24\%) eram pré-obesos e $32(31,37 \%)$ eram obesos, sendo que destes, $22(68,75 \%)$ tinham obesidade grau I, sete $(21,87 \%)$ tinham obesidade grau II e três $(9,38 \%)$ tinham obesidade grau III, segundo as diretrizes brasileiras de obesidade [10]. Observou-se predomínio da classe funcional I em 53,9\% da amostra. A FEVE média foi de $60,30 \pm 11,24 \%$, o que caracteriza $80,39 \%$ da amostra dentro dos valores de referência para a normalidade [11-13]. Dentro de cada classe funcional os valores da FEVE foram os seguintes, em mediana (intervalo interquartil): classe funcional I: 59,0 (55,0$65,0)$; classe funcional II: $56,0(48,5-61,0)$; classe funcional III: $55,5(53,0-58,0)$; e classe funcional IV: 47,5 (36,0-53,0). O Teste de Kruskal-Wallis seguido de post hoc de Dunn detectou diferenças entre os mesmos, sendo o maior valor encontrado nos pacientes com classe funcional I e o menor nos pacientes com classe funcional IV $(\mathrm{p}=0,036)$ (Figura 1).

Em relação aos fatores de risco, observou-se a prevalência de hipertensão arterial sistêmica em $82,3 \%$, dislipidemia em $57,8 \%$ e diabetes mellitus em $32,3 \%$. Estas e outras características da amostra estão apresentadas na Tabela 1.

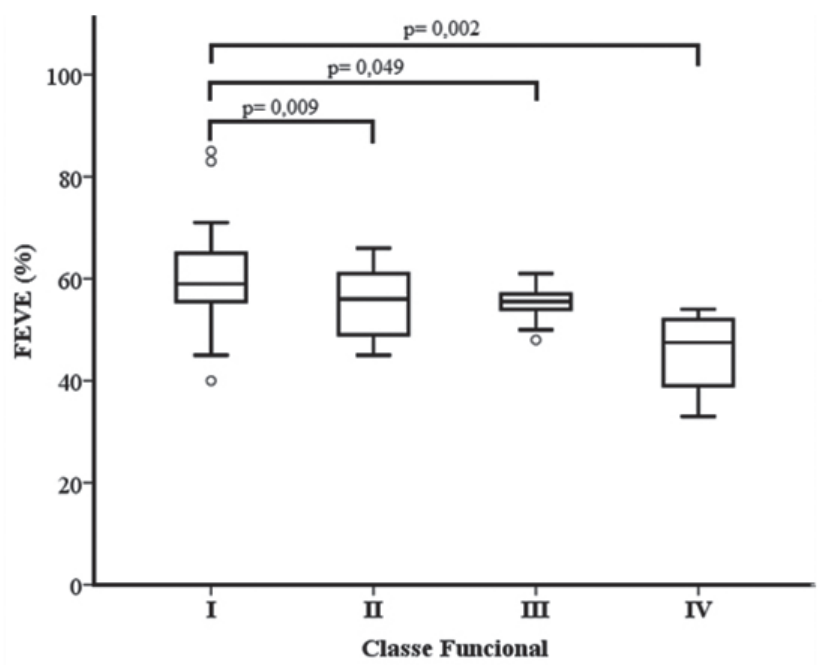

Figura 1. Diferenças entre as classes funcionais (NYHA) quanto à fração de ejeção do ventrículo esquerdo (FEVE) em pacientes com doença arterial coronariana candidatos ao programa de reabilitação cardíaca do Hospital Universitário de Santa Maria (HUSM), 2012-2016. Teste de Kruskal-Wallis seguido de post hoc de Dunn $(p=0,036)$

Tabela 1. Fatores de risco e perfil clínico referentes aos pacientes com doença arterial coronariana candidatos ao programa de reabilitação cardíaca do Hospital Universitário de Santa Maria, 2012-2016.

\begin{tabular}{lc}
\multicolumn{1}{c}{ Variáveis } & $\mathbf{n}(\%)$ \\
\hline Fatores de risco & \\
Hipertensão arterial sistêmica & $84(82,3)$ \\
Dislipidemia & $59(57,8)$ \\
Diabetes mellitus & $33(32,3)$ \\
Sedentarismo & $27(26,4)$ \\
Obesidade & $25(24,5)$ \\
Tabagismo & $17(16,6)$ \\
Classe funcional (NYHA) & \\
Classe funcional I & $55(53,9)$ \\
Classe funcional II & $33(32,3)$ \\
Classe funcional III & $10(9,8)$ \\
Classe funcional IV & $4(3,9)$ \\
Diagnóstico clínico & \\
Cardiopatia isquêmica & $70(68,6)$ \\
Prévio infarto agudo do miocárdio & $41(40,2)$ \\
Doença arterial coronariana & $13(12,7)$ \\
Valvulopatia & $12(11,7)$ \\
Procedimentos cirúrgicos & \\
Revascularização do miocárdio & $73(71,5)$ \\
Angioplastia sem stent & $56(54,9)$ \\
Angioplastia com stent & $16(15,6)$ \\
Tratamento medicamentoso & \\
Ácido acetil salicílico & $84(82,3)$ \\
Sinvastatina & $62(60,7)$ \\
Enalapril & $53(51,9)$ \\
Losartan & $22(21,5)$ \\
Mononitrato de isosorbida & $21(20,5)$ \\
Hidroclorotiazida & $19(18,6)$ \\
Furosemida & $14(13,7)$ \\
\hline &
\end{tabular}

NYHA: New York Heart Association. 


\section{DISCUSSÃO}

Este estudo demonstrou uma relação entre classe funcional e FEVE em pacientes com doença coronariana candidatos a um programa de RC. Até o limite do nosso conhecimento, este é o primeiro estudo a constatar tal desfecho nesse tipo de população. Os resultados demonstraram que indivíduos com classe funcional I apresentam FEVE maior do que aqueles com II, III e IV.

Apesar da escassez de evidências que tenham comparado a classe funcional à FEVE em pacientes com doença coronariana candidatos a um programa de RC, é importante mencionar o estudo de Mady et al. [14] que, embora tenha sido conduzido em uma população diferente da que foi objeto do presente estudo, demonstrou que a classe funcional está relacionada à capacidade de exercício e à função cardíaca em pacientes com cardiopatia chagásica. De forma semelhante, os achados do presente estudo sugerem que quanto mais avançada a classe funcional, mais comprometido o desempenho cardíaco, ou seja, menor a FEVE.

A classificação funcional estabelecida pela NYHA é um instrumento validado e de confiabilidade, o qual permite estratificar o grau de limitação imposta pela doença cardíaca na realização de atividades físicas $[15,16]$. Entretanto, sabe-se que essa classificação está sujeita à subjetividade resultante de diferentes interpretações, seja do paciente ou do observador $[17,18]$. Premissa que é retratada por Herrera et al. [19] cujo estudo destacou que mesmo pacientes que apresentavam importante redução da FEVE podiam referir ausência de sintomas, ao mesmo tempo que pacientes com FEVE preservada podiam referir intolerância ao exercício.

Apesar dessas inconsistências, a estratificação em classes funcionais tem sido utilizada como um dos critérios na avaliação de pacientes cardiopatas candidatos à RC [20]. Estudo conduzido em pacientes com insuficiência cardíaca demonstrou que a classificação funcional foi efetiva na diferenciação dos sujeitos em relação à capacidade funcional mensurada através do teste de esforço cardiopulmonar, pois diferenças no consumo máximo de oxigênio e no pulso de oxigênio foram encontradas entre as classes da NYHA [18]. Esses dados concordam com os deste estudo, no qual a efetividade da classificação funcional proposta pela NYHA foi observada através da relação inversa com a FEVE.
O teste ergométrico e a ecocardiografia são amplamente utilizados e de grande importância para a estratificação de risco em pacientes cardiopatas [21]. Os dados ecocardiográficos fornecem objetividade e estão presentes em diversos protocolos de risco cardiovascular sendo que, especificamente, a fração de ejeção já demonstra um valor preditivo de risco [22, 23].

Considerando o perfil da população estudada, constatou-se predomínio de homens de meia idade, pré-obesos, pertencentes à classe funcional I e com FEVE média de 60\%. Além disso, muitos eram hipertensos, diabéticos e dislipidêmicos e, pelos critérios de inclusão, todos apresentavam diagnóstico de cardiopatia isquêmica e haviam sido submetidos à cirurgia de revascularização do miocárdio. De forma similar, Audelin et al. [24], ao examinarem as mudanças no perfil dos indivíduos ingressantes em um programa de RC ao longo dos anos de 1996 a 2006, demostraram predomínio de pacientes idosos, do sexo masculino, com sobrepeso, diabéticos e hipertensos. Quanto à classe funcional segundo a NYHA, os achados do presente estudo assemelham-se aos citados na Diretriz de Insuficiência Cardíaca da Sociedade Europeia de Cardiologia [6], ao evidenciarem predomínio do sexo masculino em sujeitos pertencentes à classe funcional I.

No que se refere ao importante achado de pré-obesos e obesos na amostra estudada, recente estudo conduzido por Gomadam et al. [25] constatou que cerca de $40 \%$ dos pacientes ingressantes em um programa de RC eram obesos e um adicional de $40 \%$ estava com sobrepeso. De acordo com Ades et al. [26] os programas de RC com foco na prevenção secundária não podem mais ignorar o desafio do manejo da obesidade em pacientes com doença coronariana.

Este estudo apresenta algumas limitações que devem ser mencionadas: risco de viés de seleção (estudo retrospectivo) e de aferição (dados provenientes de prontuários médicos), assim como o fato do estudo ter sido realizado em um único centro.

Apesar dessas limitações, o estudo constatou que sujeitos com doença coronariana e ingressantes em um programa de reabilitação possuíam uma redução da FEVE relacionada à progressão da classe funcional de I a IV, ou seja, os sujeitos em classe funcional I apresentaram maior FEVE. Este achado fornece informações sobre as limitações impostas pela doença na capacidade de exercício e na função cardíaca do paciente, auxiliando na elaboração de um programa de treinamento físico e na otimização dos resultados clínicos após a reabilitação. 


\section{NOTAS}

\section{Apoio financeiro}

Este estudo não recebeu apoio financeiro de fontes externas.

\section{Declaração de conflito de interesses}

Os autores declaram não haver conflitos de interesses relevantes ao conteúdo deste estudo, informam ter tido acesso a todos os dados obtidos e assumem completa responsabilidade pela integridade dos resultados.

\section{REFERÊNCIAS}

1. Brasil. Ministério da Saúde, Pesquisa Nacional de Saúde 2013. Percepção do estado de saúde, estilos de vida e doenças crônicas [Internet]. Rio de Janeiro; 2013 [cited 2016 September]. Available from: http://biblioteca.ibge.gov.br/visualizacao/ livros/liv91110.pdf

2. World Health Organization (WHO). Cardiovascular diseases (CVDS) [Internet]. 2016 [cited 2016 September]. Available from: http:/www.who.int/mediacentre/factsheets/fs317/en/

3. Werf FV, Bax J, Betriu A, Blomstrom-Lundgvist C, Crea F, Falk V. Management of acute myocardial infarction in patients presenting with persistent ST - segment elevation: the Task Force on the Management of ST - Segment Elevation Acute Myocardial Infarction of the European Society of Cardiology. Eur Heart J. 2008;29(23):2909-45. https://doi.org/10.1093/ eurheartj/ehn416

4. Lear SJ, Spinelli JJ, Linden W, Brozic A, Kiess M, Frohlich JJ, Ignaszewski A. The extensive lifestyle management intervention (ELMI) after cardiac rehabilitation: a 4- year randomized controlled trial. J Am Heart Assoc. 2006;152(2):333-9. https://doi.org/10.1016/j.ahj.2005.12.023

5. Leon AS, Franklin BA, Costa F, Balady GJ, Berra KA, Stewart KJ, Thompson PD, Williams MA, Lauer MS. Cardiac Rehabilitation and Secondary Prevention of Coronary Heart Disease. An American Heart Association Scientific Statement from the Council on Clinical Cardiology (Subcommittee on Exercise, Cardiac Rehabilitation, and Prevention) and the Council on Nutrition, Physical Activity, and Metabolism (Subcommittee on Physical Activity), in Collaboration with the American Association of Cardiovascular and Pulmonary Rehabilitation. Circulation. 2005;111(3):369-76. https://doi. org/10.1161/01.CIR.0000151788.08740.5C

6. McMurray JJ, Adamopoulos S, Anker SD, Auricchio A, Böhm M, Dickstein K, Falk V, Filippatos G, Fonseca C, Gomez-Sanchez MA, Jaarsma T, Køber L, Lip GY, Maggioni AP, Parkhomenko A, Pieske BM, Popescu BA, Rønnevik PK, Rutten FH, Schwitter J, Seferovic P, Stepinska J, Trindade PT, Voors AA, Zannad F, Zeiher A; Task Force for the Diagnosis and Treatment of Acute and Chronic Heart Failure 2012 of the European Society of Cardiology, Bax JJ, Baumgartner H, Ceconi C, Dean V, Deaton C, Fagard R, Funck-Brentano C, Hasdai D, Hoes A, Kirchhof P, Knuuti J, Kolh P, McDonagh T, Moulin C, Popescu BA, Reiner Z, Sechtem U, Sirnes PA, Tendera M, Torbicki A, Vahanian A, Windecker S, McDonagh T, Sechtem U, Bonet LA, Avraamides P, Ben Lamin HA, Brignole M, Coca A, Cowburn P, Dargie H, Elliott P, Flachskampf FA, Guida GF, Hardman S, Iung B, Merkely B, Mueller C, Nanas JN, Nielsen OW, Orn S, Parissis JT, Ponikowski P; ESC Committee for Practice Guidelines. ESC guidelines for the diagnosis and treatment of acute and chronic heart failure 2012: The Task Force for the Diagnosis and Treatment of Acute and Chronic Heart Failure 2012 of the European Society of Cardiology. Developed in collaboration with the Heart Failure Association (HFA) of the ESC. Eur J Heart Fail. 2012;14(8):803-69. https://doi.org/10.1093/eurjhf/hfs 105

7. Silva JC, Rochitte CE, Júnior JS, Tsutsui J, Andrade J, Martinez EE, Moffa PJ, Menegheti JC, Kalil-Filho R, Ramires JF, Nicolau JC. Late coronary artery recanalization effects on left ventricular remodelling and contractility by magnetic resonance imaging. Eur Heart J. 2005;26:36-43. https://doi.org/10.1093/eurheartj/ehi011

8. O’Gara PT, Kushner FG, Ascheim DD, Casey DE Jr, Chung MK, de Lemos JA, Ettinger SM, Fang JC, Fesmire FM, Franklin BA, Granger CB, Krumholz HM, Linderbaum JA, Morrow DA, Newby LK, Ornato JP, Ou N, Radford MJ, Tamis-Holland JE, Tommaso JE, Tracy CM, Woo YJ, Zhao DX; CF/AHA Task Force. 2013 ACCF/AHA guideline for the management of ST-elevation myocardial infarction: executive summary: a report of the American College of Cardiology Foundation/American Heart Association Task Force on Practice Guidelines. Circulation. 2013 Jan 29;127(4):529-55. https://doi.org/10.1161/CIR.0b013e3182742c84

9. Herdy AH, López-Jimenez F, Terzic CP, Milani M, Stein R, Carvalho T; Sociedade Brasileira de Cardiologia. Diretriz Sul-Americana de Prevenção e Reabilitação Cardiovascular. Arq Bras Cardiol 2014;103(2):1-31. https://doi.org/10.5935/ abc.2014S003

10. Godoy-Matos AF, Oliveira J, Guedes EP, Carraro L, Lopes AC, Mancini MC. Diretrizes Brasileiras de Obesidade. Associação Brasileira para o Estudo da Obesidade e Síndrome Metabólica. 2009/2010; 3ạ ed. São Paulo; 2009.

11. Mann DL, Ziper DP, Libby P, Bonow RO, BraunwaldE. Braunwald's Heart Disease: A Textbook of Cardiovascular Medicine. Philadelphia: Saunders; 2015.

12. American Heart Association. Ejection fraction heart failure measurement [Internet]. [cited 2015 June]. Available from: http://www.heart.org/HEARTORG/Conditions/HeartFailure/DiagnosingHeartFailure/Ejection-Fraction-Heart-FailureMeasurement_UCM_306339_Article.jsp\#.V1i2pLsrLIU

13. Ponikowski P, Voors AA, Anker SD, Bueno H, Cleland JG, Coats AJ, Falk V, González-Juanatey JR, Harjola VP, Jankowska EA, Jessup M, Linde C, Nihoyannopoulos P, Parissis JT, Pieske B, Riley JP, Rosano GM, Ruilope LM, Ruschitzka F, Rutten FH, van der Meer P; Authors/Task Force Members; Document Reviewers. 2016 ESC Guidelines 
for the diagnosis and treatment of acute and chronic heart failure: The Task Force for the diagnosis and treatment of acute and chronic heart failure of the European Society of Cardiology (ESC). Developed with the special contribution of the Heart Failure Association (HFA) of the ESC. Eur J Heart Fail. 2016 Aug;18(8):891-975. https://doi.org/ 10.1002/ejhf.592

14. Mady C, Salemi VMC, Ianni BM, Ramires FJA, Arteaga E. Capacidade funcional máxima, fração de ejeção e classe funcional na cardiomiopatia chagásica. Existe relação entre estes índices? Arq Bras Cardiol. 2005;84(2):152-5. https:// doi.org/10.1590/S0066-782X2005000200011

15. Bocchi EA, Marcondes-Braga FG, Ayub-Ferreira SM, Rohde LE, Oliveira WA, Almeida DR, Moreira MCV, Bestetti RB, Bordignon S, Azevedo C, Tinoco EM, Rocha RM, Issa VS, Ferraz A, Cruz FD, Guimarães GV, Montera VSP, Albuquerque DC, Bacall F, Souza GEC, Neto JMR, Clausell NO, Martins SM, Siciliano A, Neto JDS, Moreira LF, Teixeira RA, Moura LZ, Beck-da-Silva L, Rassi S, Azeka E, Horowitz E, Ramires F, Simões MV, Castro RBP, Salemi VMC, Junior HV, Vila JH, Simões R, Albanesi F, Montera MW. Sociedade Brasileira de Cardiologia. III Diretriz Brasileira de Insuficiência Cardíaca Crônica. Arq Bras Cardiol. 2009;93(1 supl.1):1-71.

16. Bennett JA, Riegel B, Bittner V, Nichols J. Validity and reliability of the NYHA classes for measuring research outcomes in patients with cardiac disease. Heart Lung. 2002;31(4):262-70. https://doi.org/10.1067/mhl.2002.124554

17. Hunt SM, Baker DW, Chin MH, Cinquegrani MP, Feldman AM, Francis GS, et al. ACC/AHA Guidelines for Evaluation and Management of Chronic Heart Failure in the Adult: Executive Summary a Report of the American College of Cardiology/American Heart Association Task Force on Practice Guidelines (Committee to Revise the 1995 Guidelines for the Evaluation and Management of Heart Failure). Circulation. 2001;104(24):2296-3007. https://doi.org/10.1161/ hc4901.102568

18. Pereira DAG, Rodrigues RS, Samora GAR, Lage SM, Alencar MCN, Perreira VF, Britto RR. Capacidade funcional de indivíduos com insuficiência cardíaca avaliada pelo teste de esforço cardiopulmonar e classificação da New York Heart Association. Fisioter Pesqui. 2012;19(1):52-6. https://doi.org/10.1590/S1809-29502012000100010

19. Herrera EL, Zárate JS, Solano JF, Martínez LES, Zamudio TRP, Bautista EGB. Correlación clínica-hemodinámica de la clasificación de la NYHA/WHO en enfermos con hipertensión arterial pulmonar idiopática. Sus implicaciones en la clínica, en el tratamiento y en el pronóstico a largo plazo. Arch Cardiol Méx. 2008;78(2):148-161.

20. Santos AAS, Silva AKF, Vanderlei FM, Christofaro DGD, Gonçalves AFL, Vanderlei LCM. Analysis of agreement between cardiac risk stratification protocols applied to participants of a center for cardiac rehabilitation. Braz J Phys Ther. 2016;20(4):298-305. https://doi.org/10.1590/bjpt-rbf.2014.0159

21. American Association of cardiovascular and Pulmonary Rehabilitation. Diretrizes para reabilitação cardíaca e programas de prevenção secundária. 4⿳亠丷a ed. São Paulo: Roca; 2007.

22. Parmley WW. Position report on cardiac rehabilitation. Recommendations of the American College of Cardiology. J Am Coll Cardiol. 1986;7(2):451-3. https://doi.org/10.1016/S0735-1097(86)80526-5

23. Fletcher GF, Balady GJ, Amsterdam EA, Chaitman B, Eckel R, Fleg J, Frorlicher VF, Leon AS, Pi-a L, Rodney R, Simons-Morton DA, Williams MA, Bazzarre T. Exercise Standards for Testing and Training: A Statement for Healthcare Professionals from the American Heart Association. Circulation. 2001;104(14):1694-740. https://doi.org/10.1161/ hc3901.095960

24. Audelin MC, Savage PD, Ades PA. Changing clinical profile of patients entering cardiac rehabilitation/secondary prevention programs: 1996 to 2006. J Cardiopulm Rehabil Prev. 2008;28(5):299-306. https://doi.org/10.1097/01. HCR.0000336139.48698.26

25. Gomadam PS, Douglas CJ, Sacrinty MT, Brady MM, Paladenech CC, Robinson KC. Degree and direction of change of body weight in cardiac rehabilitation and impact on exercise capacity and cardiac risk factors. Am J Cardiol. 2016;117(4):580-4. https://doi.org/10.1016/j.amjcard.2015.11.045

26. Ades PA, Savage PD, Harvey-Berino J. The Treatment of Obesity in Cardiac Rehabilitation. Journal of cardiopulmonary rehabilitation and prevention. 2010;30(5):289-98. https://doi.org/10.1097/HCR.0b013e3181d6f9a8 C 